\title{
Induced immune tolerance of autoantigen loaded immature dendritic cells in homogenic lupus mice
}

\author{
J. Xie ${ }^{1 *}$, Y.K. Lin ${ }^{2 *}$, K. Wang ${ }^{1}$, B. Che ${ }^{1}$, J.Q. Li ${ }^{1}$, X. Xu' ${ }^{1}$, F. Han ${ }^{1}$ and \\ D.H. Liang ${ }^{1}$ \\ ${ }^{1}$ Department of Dermatology, Yangtze River Shipping General Hospital, \\ Wuhan, China \\ ${ }^{2}$ Department of Dermatology, \\ First Affiliated Hospital of Guangxi Medical University, Nanning, China \\ *These authors contributed equally to this study. \\ Corresponding author: D.H. Liang \\ E-mail: donghuiliangcn@163.com
}

Genet. Mol. Res. 13 (1): 1251-1262 (2014)

Received May 3, 2013

Accepted November 11, 2013

Published February 27, 2014

DOI http://dx.doi.org/10.4238/2014.February.27.10

\begin{abstract}
This study investigated the induced immune tolerance of autoantigen dendritic cells (imDCs) in homogenic lupus mice to support the use of dendritic cell treatment against autoimmune diseases, such as systemic lupus erythematosus. A lupus mouse was used to model based on in vitro cell culture. An immunohistochemistry assay was used to assess $\mathrm{CD} 8^{+}, \mathrm{CD}^{+}$cell ratio in mouse spleen cells. The ratio of $\mathrm{CD}^{+} \mathrm{CD} 25^{+}$cells in mouse spleen lymphocytes was detected by flow cytometry, whereas the kidney was directly measured by immunofluorescence. After the injection of mouse antigen loaded bone marrow-derived antigen imDCs with a homogenetic background, mouse nucleoprotein immune with a homogenetic background was carried out. The results were compared against the simple mouse nucleoprotein immune model with a homogenic background. The 24-h urine protein, serum antinuclear antibody and anti-ds-DNA antibodies
\end{abstract}


of the simple mouse model were lower compared to group S1. The $\mathrm{CD} 4{ }^{+} \mathrm{CD} 25^{+}$cell percentage of spleen was higher in the simple mouse model compared to group S1. In the spleen, the number of lymphocyte $\mathrm{CD} 8^{+}$cells declined, whereas the number of $\mathrm{CD} 4^{+}$cells increased. In conclusion, after autoantigen uptake, imDCs are able to induce immune tolerance to the antigen by reinfusion, which appears to prevent or mitigate systemic lupus erythematosus-like illness.

Key words: Immature dendritic cells; Lupus mice; Autoantigen; Immune tolerance

\section{INTRODUCTION}

The main manifestations of systemic lupus erythematosus (SLE) are the production of various autoantibodies, nephritis, arthritis, and other types of systemic multi-system damage, all of which lead to high mortality. Recently, dendritic cells (DCs) have been identified as the most powerful antigen-presenting cells (APCs). Furthermore, only APCs are able to activate initial immune responses (termed imDCs). ImDCs are able to 1) uptake and process antigens, 2) cause non-response and apoptosis of specific T-cells, 3) generate regulatory $\mathrm{T}$ cells, and 4) induce immune tolerance. Research of DC treatments that might effectively counteract SLE has only just begun; however, the pathogenesis of SLE and the mechanisms that some drugs act on SLE indicate a close relationship between DCs and the pathogenesis of SLE. In this experiment, imDCs loaded autoantigen ingredients were inserted in vitro into homogenic lupus mice to induce immune tolerance, and to determine whether it is possible to alleviate the symptoms of SLE.

\section{MATERIAL AND METHODS}

\section{Experimental animals}

SPF healthy BALB/c mice (6 to 8 weeks olds), weighing 18-20 g, were kept in a clean grade environment. The mice were kept at a density of 5 individuals per box. The temperature ranged from $20^{\circ}$ to $24^{\circ} \mathrm{C}$ and the relative humidity ranged from 50 to $60 \%$. Formula feed was given to the mice ad libitum, along with an unlimited water supply. The animals were provided by the Experimental Animal Center of Hubei Province, China. This study was carried out in strict accordance with the recommendations in the Guide for the Care and Use of Laboratory Animals of the National Institutes of Health, China. The animal use protocol was reviewed and approved by the Institutional Animal Care and Use Committee (IACUC) of the Yangtze River Shipping General Hospital, China.

\section{Immune models}

S1: Homologous BALB/c mouse nucleoprotein was extracted for use as an antigen. The extracted nucleoprotein and corresponding equal F's immune adjuvant were mixed to obtain a full emulsion. The antigen concentration was $1 \mathrm{mg} / \mathrm{mL}$. Mice were then immunized by injecting $50 \mu \mathrm{L}$ of the antigen intramuscularly into each hind limb tibialis anterior muscle, 
with a total dose of $100 \mu \mathrm{g}$ per mouse. Before the intramuscular injection, $100 \mu \mathrm{L} 0.5 \%$ bupivacaine was injected into the mice to cause the selective destruction of muscle fibers and regeneration of myoblasts, which would help the uptake of the antigen and produce the best immune response. The antigen injections were given over a 3-week interval, with all animals being immunized 4 times.

S2: One week before each immune injection, BALB/c mouse bone marrow-derived autoantigen loaded imDCs with a homogenetic background were input via the tail vein of mice.

S3: Mice received an injection of the same volume as the other mice, but PBS was used.

S4: This group was the control.

Nucleoprotein and imDCs were extracted from $4 \mathrm{BALB} / \mathrm{c}$ mice with homogenetic backgrounds in the S1 and S2 groups.

\section{Separation and purification of BALB/c mouse imDCs}

Following the method of Xie et al. (2009), BALB/c mice with homogenetic background were sacrificed by cervical dislocation, and placed in $75 \%$ ethanol for $5 \mathrm{~min}$. The mouse femur was removed aseptically, and placed in cold PBS. The muscle tissue was removed in a sterile console, and washed 3 times with cold PBS. The marrow cavity was repeatedly flushed until the cavity turned white. The bone marrow was washed and collected after the break-up of the erythrocytes. The collected substrate was transformed into a $1 \times 10^{6} / \mathrm{mL}$ bone marrow cell suspension by RPMI 1640 (fetal bovine serum purchased from GIBCO, Pascagoula, MS, USA) containing 10\% fetal calf serum, $20 \mathrm{ng} / \mathrm{mL}$ rmGM-CSF, and $10 \mathrm{ng} /$ mL rmIL-4 (all purchased from PeproTech, Wellesley, MA, USA). The resultant solution was seeded into 24-well plates, with $1 \mathrm{~mL}$ in each well. The plates were then placed at $37^{\circ} \mathrm{C}$ in a $5 \% \mathrm{CO}^{2}$ incubator culture for 1 day. The following day, half of the medium was changed, and a full amount of cytokines was added. Under an inverted phase contrast microscope, changes in the morphology and number of DCs were observed. The DCs exhibited a semi-suspended growth state and could be collected by repeated pipetting. The DCs from random wells were collected and pipetted into cell debris by triple-distilled water, and added to the DCs that had been cultured for 7 days. The resultant solution was co-incubated for $48 \mathrm{~h}$, and then all cells were harvested (Buelens et al., 1997). The survival rate of imDCs was assessed by AO-PI staining. Specifically, the cells were assessed under a fluorescent microscope at a 490-nm excitation light using a 510-nm grating filter, and were differentiated based on color. Endoscopic green cells represented living cells, while red cells represented dead cells.

\section{Injection of imDCs}

Autoantigen loaded imDCs of BALB/c mice with a homogenetic background were collected. The cell density was adjusted to $1 \times 10^{8} / \mathrm{mL}$.

The mouse was fixed. Then, after the preparation and disinfection of the tail skin, a proximal tourniquet ligation was made around the entire distal tail vein. Then, a 1-mL disposable syringe was used to puncture the tail vein, and the needle was withdrawn when the blood entered the needle, and the tourniquet was relaxed. An intravenous injection of $0.1 \mathrm{~mL}$ cultured cell suspension was given to mice 1 week before each nucleoprotein injection. 


\section{Protein determination}

Sulfosalicylic acid-sodium sulfate turbidimetry was applied to detect the proteins in the urine of mice for $24 \mathrm{~h}$.

\section{ELISA}

The ELIZA kit (Sigma, St. Louis, MO, USA) was used to detect mice serum antinuclear antibody (ANA) and anti-ds-DNA antibodies after the last immunization (at 22 days). The analyses were conducted using the collected blood (Xie and Lin, 2011).

\section{$\mathrm{CD}^{+} \mathrm{CD25}^{+}$cell ratios}

After the last immunization at 22 days, the mouse spleen was removed. The mice were sacrificed, and then immersed in 75\% ethanol for 1-2 min. In laminar flow, the skin was carefully cut and fixed. Then, the mouse spleen was removed from the abdominal cavity through a section of the abdomen. The aseptic technique used here should be noted.

About 4-5 mL EZ-Sep Mouse 1X lymphocyte separation medium (Dakota Biotechnology Co., Ltd.) was added to a $60-\mathrm{mm}$ Petri dish, with a fixed nylon mesh. Then, the mouse spleen was gently ground by a syringe piston to transfer the dispersed single cells into the lymphocyte separation medium through the nylon mesh. The separation liquid with suspended spleen cells was transferred to a centrifuge tube, and about $1 \mathrm{~mL}$ RPMI 1640 medium with $10 \%$ fetal calf serum was added to cover the liquid. The liquid ( $800 \mathrm{~g}$ ) was centrifuged for $30 \mathrm{~min}$, at which point the lymphocytes floated up and gathered under the upper RPMI 1640 layer. The lymphocyte layer was sucked out. Then, $10 \mathrm{~mL}$ RPMI 1640 medium was added to the lymphocytes, and $250 \mathrm{~g}$ was centrifuged for $10 \mathrm{~min}$. A volume of $500 \mu \mathrm{L}$ PBS was used to resuspend the spleen lymphocytes. A volume of $100 \mu \mathrm{L}$ of this mixture was added to each $5 \mu \mathrm{L}$ anti-mouse CD4-PE and anti-mouse CD25-FITC, and to each $5 \mu \mathrm{L}$ anti-mouse $\operatorname{lgG} 1-\mathrm{PE}$ and anti-mouse lgG2-FITC (eBioscience, San Diego, CA, USA). The solutions were incubated in the dark for $20 \mathrm{~min}$. Then, the mouse spleen lymphocytes from each group were subjected to flow cytometry to measure the ratio of $\mathrm{CD} 4^{+} \mathrm{CD} 25^{+}$cells.

\section{$\mathrm{CD8}^{+}, \mathrm{CD}^{+}$cell ratios}

After the final immunization (at 22 days), the mouse spleen was removed. The spleen was fixed in $10 \%$ formalin solution for $72 \mathrm{~h}$. After dehydration in alcohol (xylene), the spleen was embedded in paraffin, and then dewaxed, with sections of about $2 \mu \mathrm{m}$ thick being cut. A volume of $0.01 \mathrm{M}$ PBS, pH 7.4 was used to wash the sections 3 times ( 3 min for each). High pressure repaired the antigen. A volume of $2000 \mathrm{~mL} 0.01 \mathrm{M}$ citrate buffer was placed in a stainless steel pressure cooker and boiled. The slides were then placed in the solution, which was heated for a further $1.5 \mathrm{~min}$. The slides were then cooled and washed for 5 min with PBS 3 times. A volume of 3\% hydrogen peroxide solution was added to the mixture at room temperature, and left to rest for $20 \mathrm{~min}$. Then, the washing step was repeated. A volume of $50 \mu \mathrm{L}$ primary antibody (anti-mouse CD4 and CD8 was purchased from eBioscience), which 
was diluted at a ratio of 1:40 in PBS, was dropped onto each slide. The slides were subject to moist chamber incubation at $37^{\circ} \mathrm{C}$ for $2 \mathrm{~h}$. Then, the washing step was repeated again. A volume of $50 \mu \mathrm{L}$ secondary antibody (ready to use) [SuperPie Ture polymer detection (zymed packaging)] was dropped onto each slide, and incubated at room temperature for $30 \mathrm{~min}$. The washing step was repeated. $\mathrm{DAB}$ reagent was added to provide color, with the reaction requiring about $10 \mathrm{~min}$. After flushing with water, hematoxylin, and pan-blue, the slices were dried and mounted on neutral gum.

\section{Immunofluorescence detection of mouse kidney tissue}

After the final immunization (at 22 days), the kidneys were removed. The unilateral kidneys of mice from each group were collected, wrapped in gauze, and placed in an ice box to obtain quick frozen slices (using a frozen section machine; SLEE, Zeiss, Germany). Five slices were prepared from each specimen, each with a thickness of about $2 \mu \mathrm{m}$. The tissue slices were mounted onto glass slides. Then, $10 \mu \mathrm{L}$ rabbit anti-mouse IgG or IgM fluorescent antibody was dropped onto the slides (Wuhan Boster Biological Engineering Co., Ltd.) (1:40 dilution, PBS) to stain for $30 \mathrm{~min}$. The washing step was repeated and the slides were covered. The slides were then observed under a fluorescence microscope.

\section{Statistical analysis}

Statistical analysis was carried out using SPSS version 12.0 for Windows (SPSS, Chicago, IL, USA). Because all experimental groups had a normal distribution, intergroup differences were assessed using parametric statistical methods; specifically, paired independent sample $t$-tests and a one-way analysis of variance (ANOVA). Data are reported as means \pm standard deviation. Probabilities less than $5 \%(\mathrm{P}<0.05)$ were considered to be statistically significant. All experiments were repeated a minimum of 3 times.

\section{RESULTS}

\section{AO-PI staining of imDCs}

The imDC survival rate was assessed by AO-PI staining. The colors were observed under a fluorescent microscope with a 490-nm excitation light and a 510-nm grating filter. Microscopic green cells were regarded as living cells, while red cells represented dead cells. A cell survival rate of more than $90 \%$ was obtained (Figure 1).

\section{Detection of 24-h urine protein levels in mice}

Homogeneity of the variance test was performed in SPSS13.0. The homogeneity of variance was verified ( $\mathrm{W}=1.709, \mathrm{P}=0.182$ ) for the mouse urine levels. ANOVA indicated significant differences among the experimental groups $(F=33164.524, \mathrm{P}=0.000)$.

Data showed that S3 and S4 experimental groups had the lowest means for the 24-h urine protein levels, followed by the S2 group, with the S1 group having the highest levels. The SNK test was used for pairwise comparison among groups. The SNK test placed S3 and 

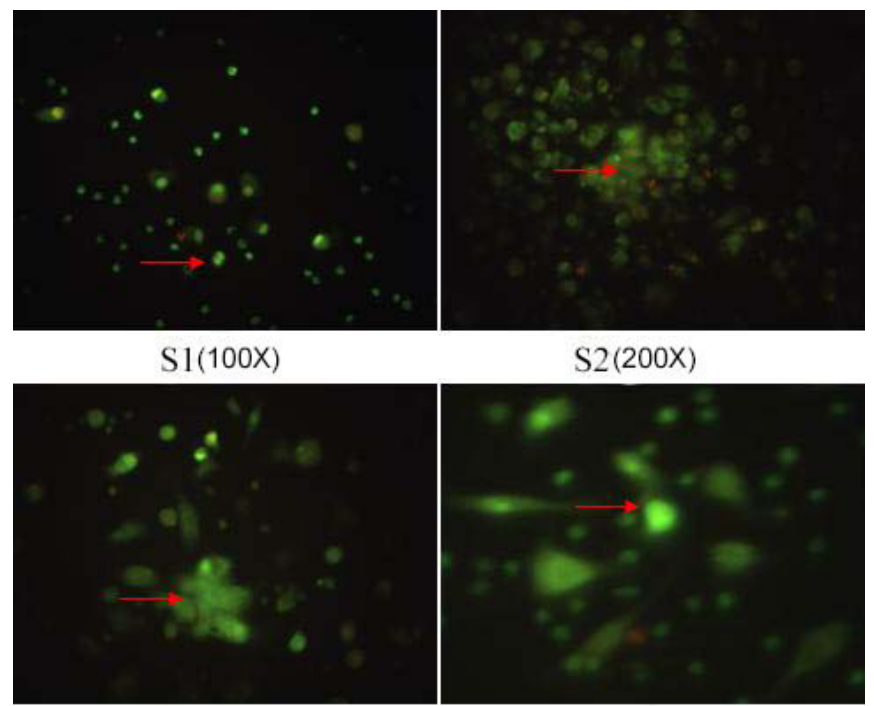

S2(200X)

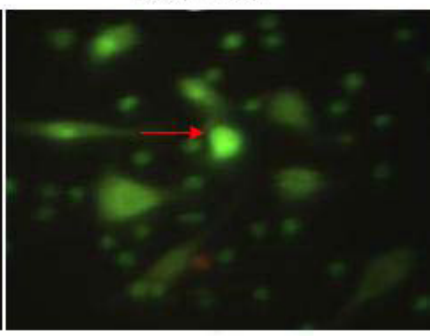

S3(200X)

S4 (400X)

Figure 1. AO-PI-staining immature dendritic cells.

S4 experimental groups in the same sub-group, while S1 and S2 were in separate sub-groups. There was no difference between S3 and S4 experimental groups. There was a difference between S1 and S2 groups. S1 and S2 groups were different compared to S3 and S4 groups. The greatest difference was obtained for the S2 group with the S3 and S4 groups (Table 1 and Figure 2).

Table 1. Twenty-four-hour urine protein in mice, anti-ds-DNA antibody, and antinuclear antibody (ANA).

\begin{tabular}{lcrr}
\hline & 24 h urine protein $(\mathrm{mg} / \mathrm{ml})$ & anti ds-DNA antibody $(\mathrm{pg} / \mathrm{ml})$ & ANA (ng/ml) \\
\hline S1 & $875.10 \pm 9.04$ & $55.52 \pm 3.04$ & $23.37 \pm 1.57$ \\
S2 & $368.50 \pm 7.00$ & $27.90 \pm 4.35$ & $17.24 \pm 1.39$ \\
S3 & $51.40 \pm 5.58$ & $9.58 \pm 1.94$ & $8.18 \pm 1.27$ \\
S4 & $49.30 \pm 4.60$ & $8.92 \pm 3.31$ & $8.16 \pm 1.39$ \\
\hline
\end{tabular}

Data are reported as means \pm SD for 10 animals in each group.

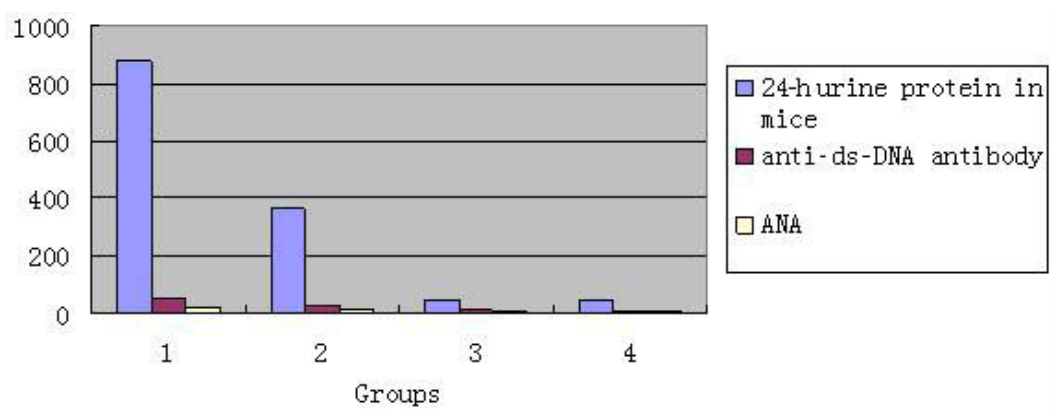

Figure 2. Twenty-four-hour urine protein in mice, anti-ds-DNA antibody, and antinuclear antibody (ANA). 


\section{Detection of the serum anti-ds-DNA antibody}

Homogeneity of the variance test was performed in SPSS13.0. The homogeneity of variance of the serum anti-ds-DNA antibody was verified $(W=1.297, \mathrm{P}=0.29)$. ANOVA indicated significant differences among the 4 groups $(F=445.93, \mathrm{P}=0.000)$.

Data showed that S3 and S4 groups had the lowest mean anti-ds-DNA antibodies, followed by S2, with S1 having the highest levels. The SNK test was used for pairwise comparison among groups. The SNK test indicated that S3 and S4 were in the same sub-group, while S1 and S2 were in 2 separate sub-groups. There was no difference between S3 and S4 groups. There was a difference between S1 and S2 groups. S1 and S2 groups were different compared to S3 and S4 groups. The greatest difference was obtained for the S2 group with S3 and S4 groups (Table 1 and Figure 2).

\section{Detection of mouse serum ANA}

Homogeneity of the variance test was performed in SPSS13.0. The homogeneity of variance verified the presence of mouse serum ANA $(W=0.14, \mathrm{P}=0.935)$. ANOVA indicated significant differences among the 4 groups $(F=278.619, \mathrm{P}=0.000)$.

Data showed that $\mathrm{S} 3$ and $\mathrm{S} 4$ groups had the lowest mean ANA of all the groups, followed by the S2 group, with the S1 group having the highest levels. The SNK test was used for pairwise comparison among groups. The SNK test indicated that S3 and S4 groups were in the same sub-group, while S1 and S2 were in 2 separate sub-groups. There was no difference between S3 and S4 groups. There was a difference between S1 and S2 groups. S1 and S2 groups were different compared to S3 and S4 groups. The greatest difference was obtained for the S2 group with the S3 and S4 groups (Table 1 and Figure 2).

\section{$\mathrm{CD}^{+} \mathrm{CD25}{ }^{+}$cell ratios of mouse spleen cells}

The $\mathrm{S} 1$ group had a lower $\mathrm{CD} 4{ }^{+} \mathrm{CD} 25^{+}$cell ratio compared to $\mathrm{S} 2, \mathrm{~S} 3$, and $\mathrm{S} 4$ groups. While the $\mathrm{S} 2$ group received additional treatment prior to the injections, it had a higher ratio compared to S3 and S4 groups (Figure 3).

\section{$\mathrm{CD4}^{+}, \mathrm{CD8}^{+}$cell ratios of mouse spleen cells (Miller et al., 2004)}

The $\mathrm{S} 1$ group had a lower $\mathrm{CD}^{+}$cell ratio compared to $\mathrm{S} 2, \mathrm{~S} 3$, and $\mathrm{S} 4$ groups. While the $\mathrm{S} 2$ group received additional treatment prior to the injections, it had a lower ratio compared to S3 and S4 groups (Figure 4).

The $\mathrm{S} 1$ group had a higher $\mathrm{CD} 8^{+}$cell ratio compared to $\mathrm{S} 2, \mathrm{~S} 3$, and $\mathrm{S} 4$ groups. While the $\mathrm{S} 2$ group received additional treatment prior to the injections, it had a higher percentage compared to S3 and S4 groups (Figure 5).

\section{Immunofluorescence of the mice kidney tissue}

The glomerulus of S1 mice had IgG and IgM immune complex deposition, along with glomerular contours. The glomerulus of S2 mice had a visible but weak fluorescence. The glomerular contours of S3 and S4 mice could not be observed, with a small amount of nonspecific weak fluorescence (Figure 6). 


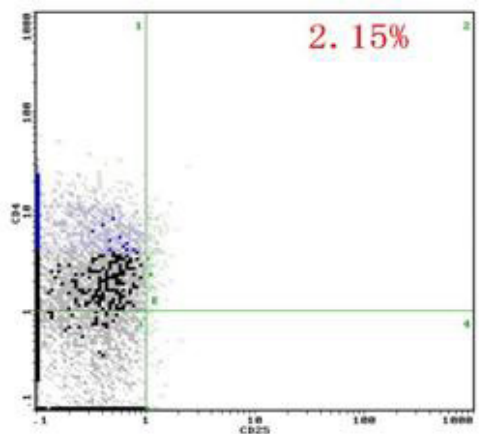

S1

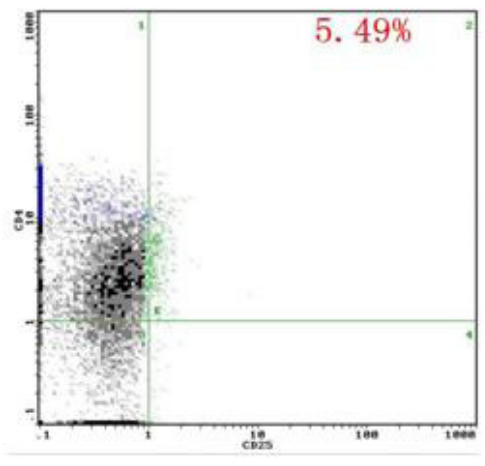

S3
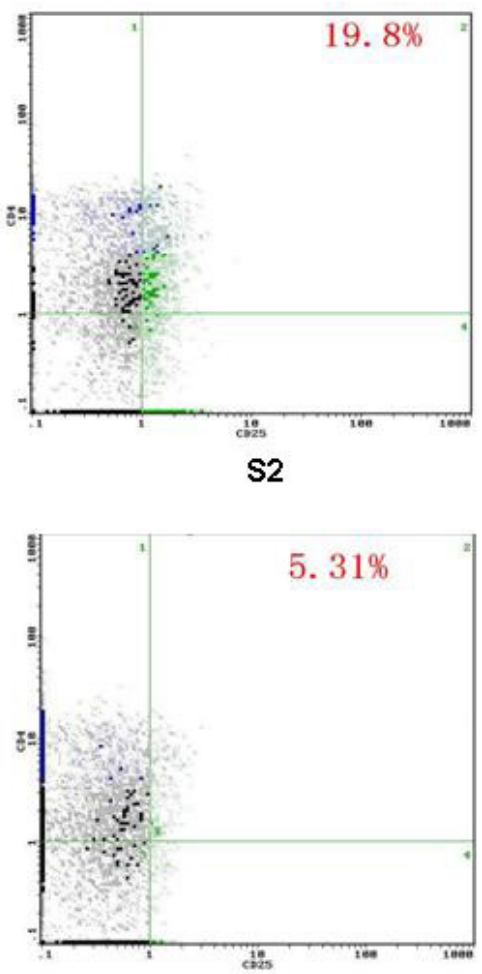

S4

Figure 3. $\mathrm{CD} 4{ }^{+} \mathrm{CD} 25^{+}$lymphocyte ratio in mouse spleen cells.
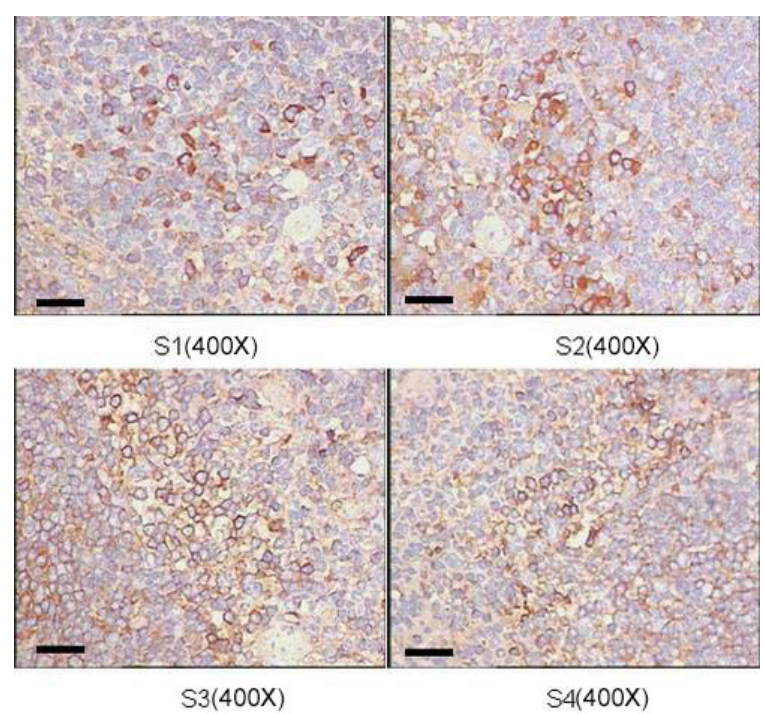

Figure 4. Changes of $\mathrm{CD}^{+}$spleen lymphocytes. 


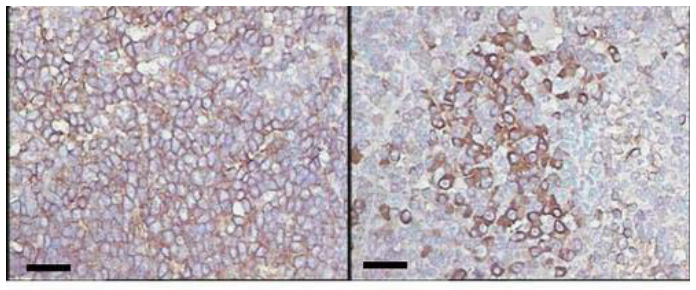

S1 (400X)

S2 (400X)

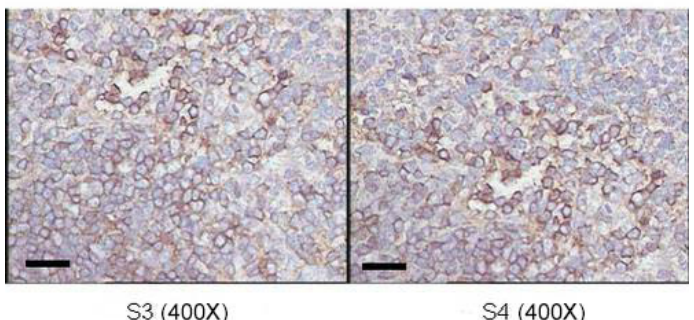

Figure 5. Changes of $\mathrm{CD} 8^{+}$spleen lymphocytes.

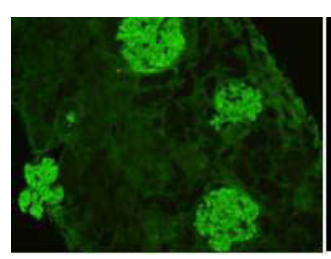

$51 \lg G(200 X)$

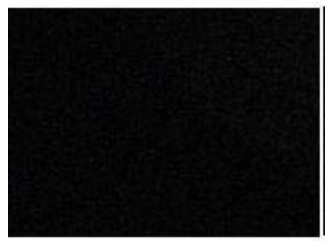

$83 \lg G(200 X)$

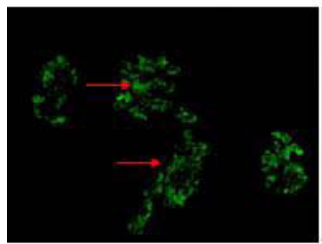

$81 \lg M(200 X)$

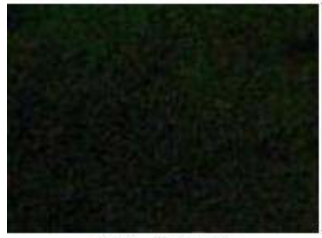

$53 \lg M(200 X)$

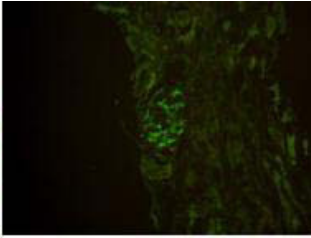

$S 2 \lg (200 X)$

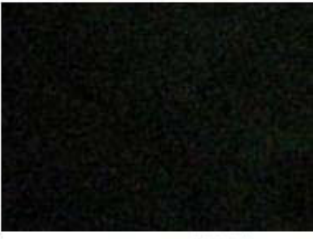

$S 4 \lg G(200 X)$

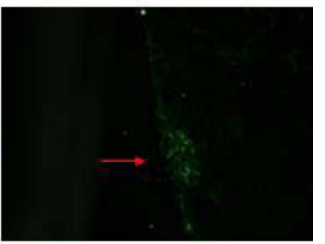

$S 2 \lg M(200 X)$

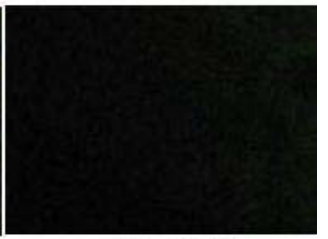

$84 \lg M(200 x)$

Figure 6. Direct immunofluorescence assay. 


\section{DISCUSSION}

Recent research has confirmed the important role of DCs in the immune system, along with their ability to suppress tumors; hence, research in this area has become a major focus of immunology studies and related fields. In addition, the immune tolerance of imDCs is also receiving major attention. In-depth studies of DCs have indicated the presence of a complex and sophisticated adjustment system that forms part of the major immunomodulatory system (Banchereau and Steinman, 1998). DCs have several major characteristics. First, the DC-mediated immune process is a precise and rigorous process, which involves the timely and accurate migration of DCs to draining lymph nodes, forming the basis of the body's normal immune response. The presence of undesired factors, lead to abnormal migration, producing a pathological immune response. Second, DCs are regulated by cytokines, chemokines, and chemo-attractants and their corresponding ligands, in addition to hormones, proteins, and bacterial products. DCs serve as immune promoters or inhibitors, and are involved in various synergistic or antagonistic activities. Under certain conditions, DCs have contradictory effects. Third, DC adjustment is very complex. For instance, while many trials and studies have demonstrated that many factors affect the activity of DCs, detailed information about the involved mechanisms remains unclear. Fourth, DCs participate in the rejection of some tumors, or escape processes. Recent research has demonstrated the role of DCs in autoimmune diseases; however, the exact mechanisms require further study. Therefore, future research should focus on elucidating the mechanisms of action of various DC factors, including their synergistic and antagonistic modes, pathways, and mechanisms, as well as obtaining details about how their actions are adjusted under different physiological conditions. For instance, the mechanisms of action under different pathological states require clarification to better explain the impacts of various harmful factors on DCs.

Clinical research that focuses on how to treat a variety of immune diseases and a variety of infections through the promotion or suppression of DCs is also vital. Studies to generate vaccines to obtain immunity against tumors have been initiated using the biological functions and migration characteristics of DCs. At present, imDCs have become the major focus for addressing autoimmune diseases (Bluestone et al., 2007). ImDCs express low levels of major histocompatibility complex MHC II molecules, co-stimulatory molecules, and adhesion molecules. Each DC expresses about $1 \times 10^{6} \mathrm{MHC}$ II molecules. In vitro studies of DCs in mixed lymphocyte culture (MLC) exhibit a weak capacity to stimulate a mixed lymphocyte reaction (MLR). In addition, DCs exhibit a strong capacity for antigen uptake and processing, without altering the ability of antigens. After the uptake of antigens or after a certain stimulus (i.e., inflammatory signals), imDCs differentiate and mature, with each cell expressing about 7 x $10^{6} \mathrm{MHC}$ II molecules. This phenomenon significantly enhances co-stimulatory molecules and adhesion molecules. In vitro MLC has a strong stimulatory ability on MLR; however, the uptake and processing ability of antigens is significantly reduced (Henrickson et al., 2008). It was first confirmed that DCs induce immune tolerance in the central self-tolerance. Existing studies show that the injection of allogeneic DCs into the thymus of newborn mice induces immune tolerance (Michaels et al., 2005). Similar results were obtained in studies of bone marrow chimeras and transgenic mice. The immune tolerance obtained from thymic allogeneic antigen injection appears to depend on DCs in the thymus. In addition, donor bone marrow-derived DCs combined with allogeneic peptide-pulsed thymic injection might induce the organ immune tolerance of anti-donor lymphocyte-positive recipients (Ali et al., 2000). The body's autoantigens are able to reach the thymus through the blood circulation. The central 
immune tolerance of T-cells is induced by the presentation of thymus DCs. Peripheral blood DCs might also carry foreign antigens, which enter the thymus and play a role in the central tolerance of certain foreign antigens in the thymus. The peripheral tolerance effects of DCs are usually mediated by imDCs and might also depend on the functioning of a certain DC subset. ImDCs show a low expression of co-stimulatory molecules, and (like autoantigens) cannot activate T-cells when in the peripheral lymphoid tissues. However, DCs might also induce Tcell anergy or cause auto-tolerance (Gonzalez-Rev et al., 2006).

ImDCs and inhibitory DC subsets also induce regulatory/suppressor T-cells to clear immune reactivity T-cells. ImDCs also produce IL-10 and TGF- $\beta$ cytokines to suppress immune reactive T-cells directly, enabling immune tolerance (den Haan et al., 2007). ImDCs capture antigens and place them on the effector cell surface (MHC molecules), in addition to engulfing and processing apoptotic cells. Apoptotic cells are effectively captured by DCs, resulting in DCs inducing a weakened inflammatory response function, which facilitates antigen-specific unresponsiveness. The uptake of apoptotic cells does induce inflammation or their transformation into mature cells. In addition, DCs are able to handle and present polypeptides from apoptotic cells (Selenko-Gebauer et al., 2003). In homeostasis, DCs are targeted towards infusing the apoptotic leukocytes of the donor; hence, the donor might be able to produce regulatory DCs selectively in vivo, within the framework of a cell transplantation therapy program. However, donor leukocytes must be obtained that are in homeostasis, with the injection being carried out in the first few days. This type of program could be applied to a living donor organ transplant (Ferguson et al., 2002).

In animal models, bone marrow cells receive a low-dose of GM-CSF, which cultivates experimental myeloid imDCs and induces the production of antigen-specific $\mathrm{T}$ cell hyporesponsiveness in vitro. Selenko-Gebauer et al. (2003) found that, in the imDC membrane, B7 family molecules PD-L1 and PD-L2 provide a weak co-stimulatory signal that induces T cell anergy. In such cases, cytokine-stimulated-cultured donor myeloid imDCs in vitro are transplanted into the recipient's body to extend graft survival time. Th1 and Th2 cells produce mutual inhibitory cytokines (Billiard et al., 2006). ImDCs secrete IL-10, TGF- $\beta$, and CTLA-Ig to induce Th1/Th2 imbalance (Bank, 1988; Renkl et al., 2005). ImDCs of the IL-10 loaded plasmid immune rat are able to downregulate MHC class II molecules and co-stimulatory molecules. The infusion of such imDCs to autoimmune myocarditis rats enhances myocardial function, by causing peripheral blood T cells to become more skewed towards skewed Th2 (Murdoch et al., 2004; Kim et al., 2005). Another study showed that bone marrow imDCs of IL-10 immunized BALB/c mice reduce the ability of DCs to stimulate Th1 and Th2 proliferations, but do not affect the Th2 immune response (O'Connell et al., 2002).

In the current experiment, the bone marrow cells of $\mathrm{BALB} / \mathrm{c}$ mice with autologous were lysed to extract the self-antigens of the mice as an antigen substance. Immunohistochemical and electron microscopy observations after DCs self-antigen loading confirmed that DCs are able to present self-antigens. Nucleoprotein immune was carried out after injecting the mouse bone marrow-derived imDCs loaded mouse antigen with the same genetic background. The 24-h urinary protein levels were lower for nucleoprotein immune mice compared to the simple lupus mouse (Satoh et al., 2000; Decker et al., 2005). Serum ANA and anti-ds-DNA antibody levels were lower in the former compared to the latter. In spleen cells, the $\mathrm{CD} 4{ }^{+} \mathrm{CD} 25^{+}$cell ratio was higher in the S2 compared to the S1. In the spleen lymphocytes, the $\mathrm{CD} 8^{+}$lymphocyte levels were lower in the S2 compared to the S1, whereas $\mathrm{CD} 4^{+}$lymphoid cell levels were higher. The observed changes after autoantigen uptake, prompted imDCs to induce immune tolerance through reinfusion, which might potentially prevent or mitigate SLE-like illnesses. This study 
has identified the basal mechanisms of DC vaccines, which might be used to treat and prevent SLE.

\section{ACKNOWLEDGMENTS}

Research supported by the Yangtze River Shipping administration (grant \#201110024) and the Wuhan City Bureau of Health (grant \#WH11D23).

\section{REFERENCES}

Ali A, Garrovillo M, Jin MX, Hardy MA, et al. (2000). Major histocompatibility complex class I peptide-pulsed host dendritic cells induce antigen-specific acquired thymic tolerance to islet cells. Transplantation 69: 221-226.

Banchereau J and Steinman RM (1998). Dendritic cells and the control of immunity. Nature 392: 245-252.

Bank HL (1988). Rapid assessment of islet viability with acridine orange and propidium iodide. In Vitro Cell Dev. Biol. 24: $266-273$.

Billiard F, Litvinova E, Saadoun D, Djelti F, et al. (2006). Regulatory and effector T cell activation levels are prime determinants of in vivo immune regulation. J. Immunol. 177: 2167-2174.

Bluestone JA, Thomson AW, Shevach EM and Weiner HL (2007). What does the future hold for cell-based tolerogenic therapy? Nat. Rev. Immunol. 7: 650-654.

Buelens C, Verhasselt V, De Groote D, Thielemans K, et al. (1997). Interleukin-10 prevents the generation of dendritic cells from human peripheral blood mononuclear cells cultured with interleukin-4 and granulocyte/macrophagecolony-stimulating factor. Eur. J. Immunol. 27: 756-762.

Decker P, Singh-Jasuja H, Haager S, Kötter I, et al. (2005). Nucleosome, the main autoantigen in systemic lupus erythematosus, induces direct dendritic cell activation via a MyD88-independent pathway: consequences on inflammation. J. Immunol. 174: 3326-3334.

den Haan JM, Kraal G and Bevan MJ (2007). Cutting edge: Lipopolysaccharide induces IL-10-producing regulatory CD4+ T cells that suppress the CD8+ T cell response. J. Immunol. 178: 5429-5433.

Ferguson TA, Herndon J, Elzey B, Griffith TS, et al. (2002). Uptake of apoptotic antigen-coupled cells by lymphoid dendritic cells and cross-priming of CD8 $(+) \mathrm{T}$ cells produce active immune unresponsiveness. J. Immunol. 168: 5589-5595.

Gonzalez-Rey E, Chorny A, Fernandez-Martin A, Ganea D, et al. (2006). Vasoactive intestinal peptide generates human tolerogenic dendritic cells that induce CD4 and CD8 regulatory T cells. Blood 107: 3632-3638.

Henrickson SE, Mempel TR, Mazo IB, Liu B, et al. (2008). T cell sensing of antigen dose governs interactive behavior with dendritic cells and sets a threshold for T cell activation. Nat. Immunol. 9: 282-291.

Kim SH, Lechman ER, Bianco N, Menon R, et al. (2005). Exosomes derived from IL-10-treated dendritic cells can suppress inflammation and collagen-induced arthritis. J. Immunol. 174: 6440-6448.

Michaels MA, Kang HK, Kaliyaperumal A, Satyaraj E, et al. (2005). A defect in deletion of nucleosome-specific autoimmune T cells in lupus-prone thymus: role of thymic dendritic cells. J. Immunol. 175: 5857-5865.

Miller MJ, Safrina O, Parker I and Cahalan MD (2004). Imaging the single cell dynamics of CD4+ T cell activation by dendritic cells in lymph nodes. J. Exp. Med. 200: 847-856.

Murdoch TB, McGhee-Wilson D, Shapiro AM and Lakey JR (2004). Methods of human islet culture for transplantation. Cell Transplant. 13: 605-617.

O'Connell PJ, Li W, Wang Z, Specht SM, et al. (2002). Immature and mature CD8alpha+ dendritic cells prolong the survival of vascularized heart allografts. J. Immunol. 168: 143-154.

Renkl AC, Wussler J, Ahrens T, Thoma K, et al. (2005). Osteopontin functionally activates dendritic cells and induces their differentiation toward a Th1-polarizing phenotype. Blood 106: 946-955.

Satoh M, Richards HB, Shaheen VM, Yoshida H, et al. (2000). Widespread susceptibility among inbred mouse strains to the induction of lupus autoantibodies by pristane. Clin. Exp. Immunol. 121: 399-405.

Selenko-Gebauer N, Majdic O, Szekeres A, Hofler G, et al. (2003). B7-H1 (programmed death-1 ligand) on dendritic cells is involved in the induction and maintenance of T cell anergy. J. Immunol. 170: 3637-3644.

Xie J and Lin YK (2011). A mouse model of lupus induced by nucleoprotein with the same genetic background. J. Clin. Rehabilitative Tissue Eng. Res. 15: 8613-8616.

Xie J, Lin YK and Luo H (2009). The influence of swallowing autoantigen on differentiation, development and function of the bone marrow-derived DC in vitro. Chin. J. Immunol. 9: 774-779. 